\title{
CONCENTRACIÓN ANESTÉSICA DEL EUGENOL EN PECES ESCALARES (Pterophyllum scalare)
}

\author{
Anesthetic Concentration of Eugenol in Angelfish (Pterophyllum scalare) \\ Laura Millán-Ocampo ${ }^{1}$, Anyi Torres-Cortés ${ }^{1}$, Gira Alejandra Marín-Méndez ${ }^{1}$, \\ Wilson Ramírez-Duart ${ }^{2}$, Mónica Andrea Vásquez-Piñeros², \\ Iang Schroniltgen Rondón-Barragán ${ }^{1,2,3}$
}

\section{RESUMEN}

El eugenol (aceite de clavo) es un compuesto que ha sido ampliamente utilizado como anestésico en peces debido a sus cortos tiempos de inducción y recuperación. El objetivo del estudio fue determinar la concentración anestésica efectiva del eugenol en 40 peces escalares (Pterophyllum scalare) bajo condiciones de laboratorio. Los animales fueron expuestos a cuatro concentraciones de la solución anestésica (20, 30, 40 y 50 $\mathrm{mg} / \mathrm{L}$ de eugenol) y las observaciones se hicieron en los estadios de inducción a la anestesia (I, II, III) y recuperación (I, II, III, IV). Los animales expuestos a las mayores concentraciones de eugenol (40 y $50 \mathrm{mg} / \mathrm{L}$ ) entraron rápidamente en inducción; sin embargo, fueron los que más tardaron en recuperarse $(\mathrm{p}<0.05)$. En una concentración de 20 $\mathrm{mg} / \mathrm{L}$ se induce anestesia ligera en peces escalares (estadio I de anestesia), en tanto que una concentración de $50 \mathrm{mg} / \mathrm{L}$ se induce un estadio de anestesia avanzado (III). La concentración anestésica más efectiva fue de $40 \mathrm{mg} / \mathrm{L}$ de eugenol para alevinos de peces escalares.

Palabras clave: peces ornamentales, anestesia, eugenol, escalares

\section{AbSTRACT}

Eugenol (clove oil) has been widely used as an anaesthetic in fishes due to low induction and recovery times. The aim of this study was to assess the anaesthetic concentration of eugenol in 40 angel fish (Pterophyllum scalare) under laboratory conditions. The animals were exposed to four concentrations of eugenol (20, 30, 40 and $50 \mathrm{mg} / \mathrm{L}$ ). Observations of the anaesthetics induction (phase I, II, and III) and recovery (phase I, II, III, and IV) were registered. The animals exposed to the higher concentrations of eugenol (40 and $50 \mathrm{mg} / \mathrm{L})$ reached in a short time the first induction anaesthetic stage but had the longer recovery time $(\mathrm{p}<0.05)$. The $20 \mathrm{mg} / \mathrm{L}$ concentration of eugenol induce

${ }^{1}$ Inmunología y Fisiopatología Animal (IFA), Facultad de Medicina Veterinaria y Zootecnia, Departamento de Sanidad Animal, Universidad del Tolima, México

2 Sanidad de Organismos Acuáticos, Instituto de Acuicultura de los Llanos, Universidad de los Llanos, Colombia

${ }^{3}$ E-mail: isrondon@ut.edu.co.URL: http://ifamvzut.es.tl/ 
light anaesthetic stage in angel fish (phase I) while the $50 \mathrm{mg} / \mathrm{L}$ concentration induce an advanced stage of anaesthetic (III). The most effective concentration was $40 \mathrm{mg} / \mathrm{L}$ of eugenol for angel fish.

Key words: ornamental fish, anesthesia, eugenol, angelfish

\section{INTRODUCCIÓN}

Los anestésicos constituyen una herramienta importante en piscicultura para reducir los niveles de estrés, las lesiones y la mortalidad durante y después del manejo y el transporte. Los anestésicos utilizados tradicionalmente en piscicultura incluyen tricaína metanosulfato (MS-222), quinaldina y 2fenoxietanol (Façanha y Gomes, 2005); sin embargo, presentan limitantes dado su alto costo y toxicidad asociada (Velisek et al., 2006). Así, se ha demostrado que el MS-222 eleva el nivel de cortisol en plasma de peces completamente anestesiados (Coyle et al., 2004); por otra parte, Molinero y González (1995) y Hill y Forster (2004) encontraron que estos anestésicos incrementan la respuesta al estrés $y$, por ende, aumentan la actividad metabólica.

El eugenol [2-methoxy-4-2-(2propenyl)-phenol], también llamado aceite de clavo, se obtiene de hojas, flores y tallos de árboles de clavo (Eugenia caryophyllata Thunberg y Eugenia aromatica Baill) (Soto y Burhanuddin, 1995), y es ampliamente utilizado en acuicultura como anestésico en peces y en moluscos (Keene et al., 1998; Woody et al., 2002; Seol et al., 2007), especialmente para la sedación y anestesia a bajas concentraciones. Es de fácil adquisición, económico, ambientalmente amigable, y seguro para los operarios y los peces (Soto y Burhanuddin, 1995; Iversen et al., 2003; Cunha y Rosa, 2006). Este compuesto ha sido clasificado por la US Food and Drug Administration como seguro para el humano cuando no se excede de $1500 \mathrm{mg} / \mathrm{kg}$ (Anderson et al., 1997).
Varios autores han reportado tiempos cortos de inducción y de recuperación con eugenol (Munday y Wilson, 1997; Woody et al., 2002); sin embargo, el tiempo de recuperación es mayor en comparación con anestésicos tradicionales, pero es efectivo a concentraciones más bajas que las requeridas con MS-222, benzocaína y 2-fenoxietanol (Munday y Wilson, 1997; Sladky et al., 2001; Ackerman y Bellwood, 2002).

Woody et al. (2002) determinaron que el eugenol es útil como anestésico en el salmón rojo (Oncorhynchus nerka), obteniendo un estadio III de inducción anestésica con $50 \mathrm{mg} / \mathrm{L}$ y anestesia quirúrgica con $80 \mathrm{mg} / \mathrm{L}$ en menos de tres minutos y con recuperación en menos de 10 minutos, y sin casos de mortalidad ante exposiciones de 15 minutos. Además, encontraron mayores tiempos de inducción a la anestesia para peces más grandes, aunque el tamaño del pez no influyó en la recuperación de la anestesia. Asimismo, Cunha y Rosa (2006) reportaron concentraciones de 20 a $60 \mathrm{mg} / \mathrm{L}$ de eugenol efectivas para la inmovilización de siete especies de peces tropicales de arrecife, con periodos de inducción cortos $(<2 \mathrm{~min})$ y tiempos de recuperación de 2 a 4 minutos que estuvieron directamente relacionados con la concentración del anestésico. De este modo, se ha hallado una correlación positiva entre la concentración del eugenol y la reducción del tiempo de inducción y prolongación de la recuperación (Soltani et al., 2004; Cunha y Rosa, 2006).

En relación a la liberación del cortisol, se encontró que tanto el eugenol (50 a 500 $\mathrm{mg} / \mathrm{L}$ ) como el 2-fenoxietanol (510 mg/L) no impiden su liberación ni inducen alteraciones 
hematológicas en dorada (Sparus aurata) y trucha (Oncorhynchus mykiss) (Tort et al., 2002), evidenciando su potencial como anestésico seguro.

El eugenol, así como otros aceites esenciales, induce un incremento agudo de la concentración de $\mathrm{Ca}^{++}$intracelular en células que expresan el receptor de potencial transitorio, subfamilia A, miembro 1 o TRPA1 (Bandell et al., 2004). De este modo, la sensación de calentamiento experimentada frecuentemente con la aplicación de aceites esenciales, entre ellos el mentol, ocurre en parte por activación de receptores de frío lesivo. El eugenol puede, además, activar el receptor de potencial transitorio, subfamilia M, miembro 8 o TRPM8 (Harris, 2006).

El objetivo del presente estudio fue determinar la concentración de eugenol (aceite de clavo) en peces escalares (Pterophyllum scalare) que permitan estadios anestésicos que garanticen su inmovilización bajo condiciones de laboratorio.

\section{MATERIALES Y MéTodos}

\section{Localización}

Los experimentos se realizaron en el laboratorio de bioensayos del Grupo de Investigación en Sanidad de Organismos Acuáticos, del Instituto de Acuicultura de los Llanos (IALL), Universidad de los Llanos, Meta - Colombia.

\section{Características del Estudio}

Se utilizaron 40 peces escalares (Pterophyllum scalare) pertenecientes al IALL, clínicamente sanos, de $6 \pm 2 \mathrm{~g}$ de peso, $5.14 \pm 0.63 \mathrm{~cm}$ de longitud estándar, mantenidos en acuarios de vidrio, y alimentados con concentrado comercial ( $30 \%$ de proteína) ad libitum. Los procedimientos fueron de carácter no invasivo y aprobados por el Comité de Ética del Instituto de Investigaciones de la Orinoquia Colombiana - IIOC.
Se utilizó el Eugenol ${ }^{\circledR}$ (The J Bird Moyer Co., Inc, EEUU), (4-alil, 2-metoxifenol, densidad $1.04 \mathrm{~g} / \mathrm{mL}$ ), como anestésico siguiendo los lineamientos de Munday y Wilson (1997). Para esto, se realizó una dilución de 1:9 con etanol (Merck, Germany, 99.9\%) logrando una concentración final de $100 \mathrm{mg} / \mathrm{mL}$ de eugenol.

Los animales fueron sometidos a la solución experimental en acuarios con capacidad para $10 \mathrm{~L}$ con aireación constante sin filtro. Se monitoreó oxígeno, temperatura (con oxímetro Hach Portable LDO ${ }^{\mathrm{TM}}$ HQ10 Dissolved Oxygen Meter $^{\circledR}$, EEUU), $\mathrm{pH}$ (con pH-metro Pinpoint $\mathrm{pH}$ Monitor ${ }^{\circledR}$, American Marine Inc., EEUU), conductividad (con sonda multiparamétrica YSI556), dureza y alcalinidad.

\section{Diseño Experimental}

Se establecieron cuatro concentraciones de eugenol (I: 20, II: 30, III: 40, y IV: 50 $\mathrm{mg} / \mathrm{L}$ ) en base a ensayos preliminares. El anestésico fue disuelto en el agua del acuario cinco minutos antes de la inmersión de cada animal y se renovó la solución anestésica para el experimento con cada animal (réplica). En cada una de las concentraciones evaluadas se utilizaron 10 animales. Los estadios de anestesia (Cuadro 1) fueron determinados de acuerdo con ensayos preliminares (Soto y Burhanuddin, 1995; Keene et al., 1998; Walsh y Pease, 2002; Woody et al., 2002; Cunha y Rosa, 2006). Una vez alcanzada la última fase de inducción, los animales fueron inmediatamente transferidos a acuarios con agua libre de anestésico para evaluar la recuperación de la anestesia de acuerdo a los descrito por Walsh y Pease (2002) y Keene et al. (1998) (Cuadro 1).

La evaluación del efecto anestésico se realizó en exposiciones individuales para facilitar la observación de los diferentes planos de anestesia. Se realizó el registro en video. El tiempo fue medido mediante un cronómetro de precisión (CASIO, Stopwatch ${ }^{\circledR}$ HS-3, Japón). 
Cuadro 1. Características del estado de anestesia y recuperación de Pterophyllum scalare inmersos en eugenol

\begin{tabular}{clcl}
\hline $\begin{array}{c}\text { Estadio de } \\
\text { an estesia }\end{array}$ & Característica & $\begin{array}{c}\text { Estadio de } \\
\text { recuperación }\end{array}$ & Característica \\
\hline I & $\begin{array}{l}\text { Pérdida parcial del eje de } \\
\text { nado }\end{array}$ & I & $\begin{array}{l}\text { Recuperación del movimiento } \\
\text { opercular } \\
\text { Recuperación del movimiento } \\
\text { de aletas }\end{array}$ \\
II & Pérdida total de eje de nado & II & $\begin{array}{l}\text { Recuperación total del eje de } \\
\text { nado }\end{array}$ \\
III & Colapso medular & III & $\begin{array}{l}\text { Recuperación del reflejo de } \\
\text { huida }\end{array}$ \\
\hline
\end{tabular}

El etanol $(99.9 \%)$ se utilizó como diluyente del anestésico, de modo que se expusieron los animales a la concentración más alta de etanol utilizada en las cuatro concentraciones de anestésicos $(0.4 \mathrm{ml}$ de etanol por litro) durante 10 minutos, con el fin de determinar si la exposición al etanol generó alguna de las características asociadas con la anestesia de los peces. Las condiciones experimentales (i.e. volumen de agua, calidad de agua) fueron las mismas a las descritas para la exposición al anestésico.

\section{Análisis Estadístico}

Se utilizó un diseño experimental completamente aleatorizado efecto fijo balanceado con la prueba de ANOVA y MANOVA [Yij $\left.=\mu+\frac{1}{100} \mathrm{i}+\mathrm{gi}(\mathrm{I})\right]$, donde $\mu$ es la media, $\frac{1}{100} \mathrm{i} \mathrm{el}$ efecto tratamiento y gj(i) el error experimental. La variable respuesta fue el tiempo expresado en segundos.

Se utilizaron las pruebas de contraste de Tukey y análisis canónico de índice ortogonal con un $95 \%$ de confiabilidad. Se validaron los supuestos del modelo y se empleó un análisis descriptivo exploratorio unidimensional para hallar media, desviación y coeficiente de variación. Los datos fueron transformados como $\log _{10}$ para el cumplimiento de los supuestos estadísticos. Los datos son presentados como promedios y error estándar de la media (ESM). Los resultados fueron analizados mediante el software SAS/ $\mathrm{STAT}^{\circledR} \mathrm{v} 9.0$ y graficados mediante SPSS v 19 para Windows.

\section{Resultados}

No se presentaron casos de mortalidad en los individuos inducidos a anestesia con eugenol, ni estadios anestésicos en los animales expuestos a la mayor concentración del diluyente etanol. En todas las concentraciones, antes de la fase II de inducción de la anestesia, los animales evidenciaron episodios de nado rápido y de corta distancia (hiperactividad inicial) por periodos cortos de tiempo. Este carácter explosivo de nado no se evidenció en las fases de recuperación.

No hubo diferencias estadísticas entre tratamientos para los tiempos de inducción I y II de anestesia; sin embargo, durante el tercer estadio de inducción se registró un menor tiempo para las concentraciones de $40 \mathrm{y}$ $50 \mathrm{mg} / \mathrm{L}$ en relación 20 y $30 \mathrm{mg} / \mathrm{L}(\mathrm{p}<0.05$, Fig. 1). 

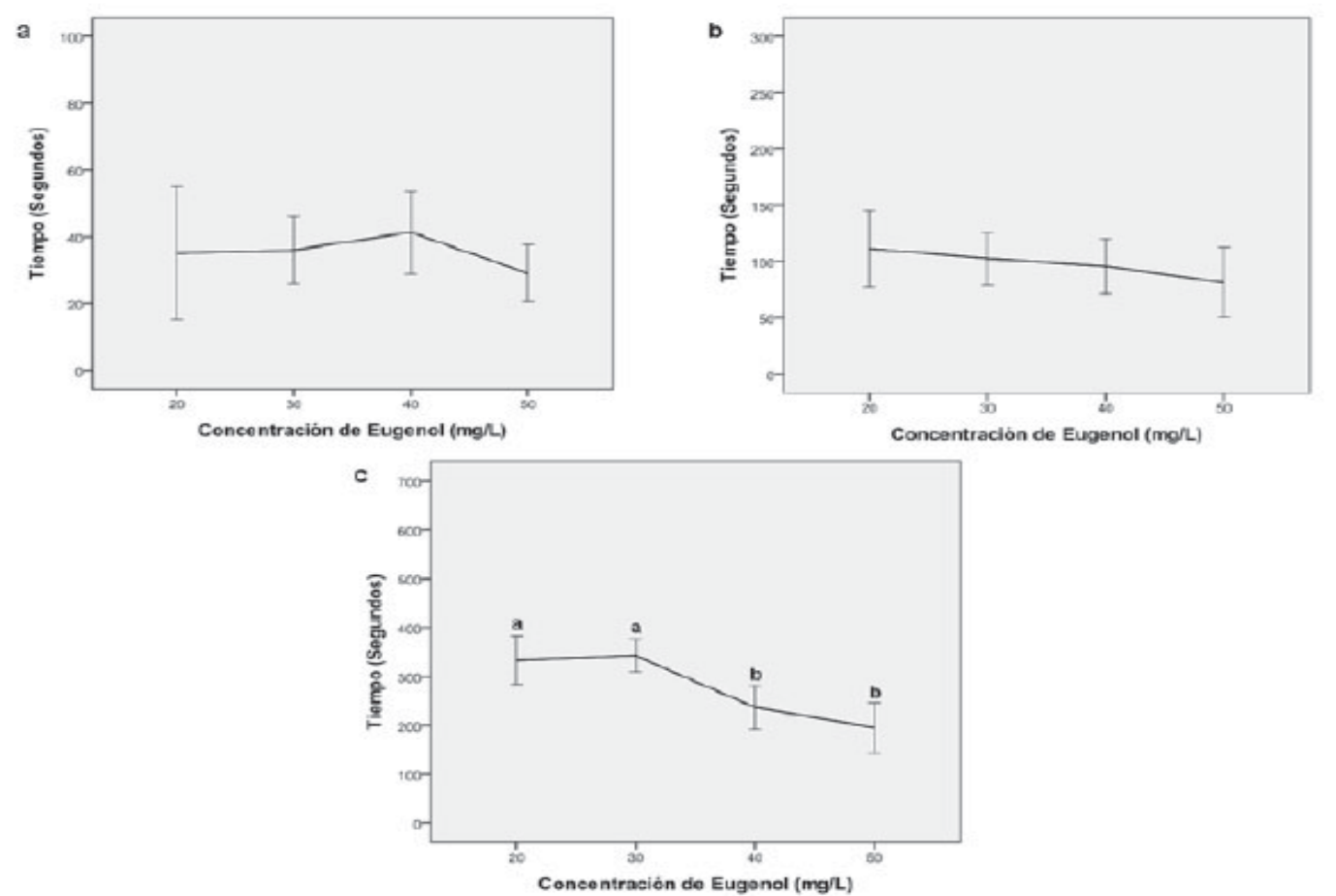

Figura 1. Tiempos de inducción a diferentes concentraciones de eugenol en peces escalares (Pterophyllum scalare). Planos de anestesia: (a) Inducción I, (b) Induces denotan diferencias estadísticas $(p<0.05)$
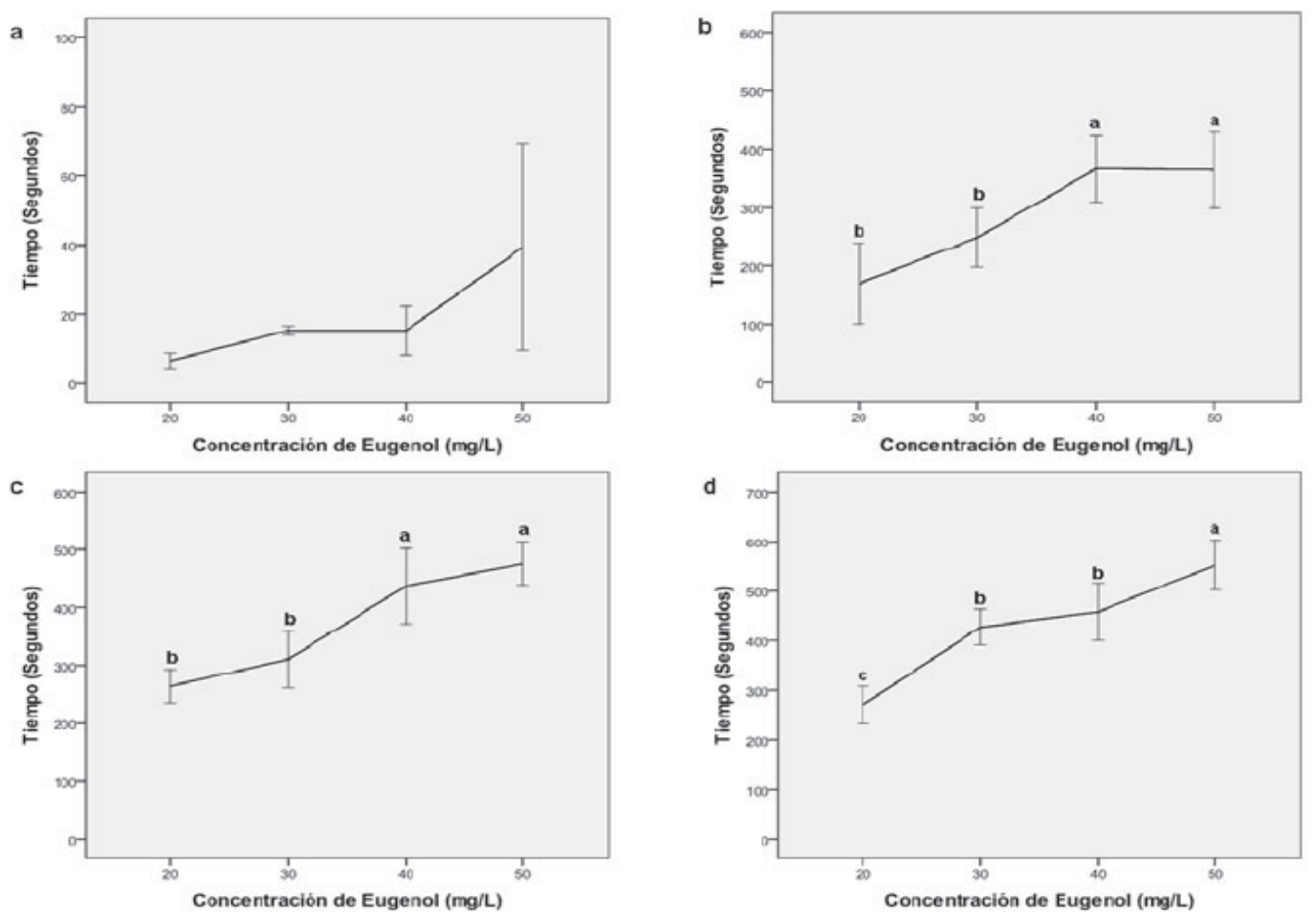

Figura 2. Tiempos de recuperación a diferentes concentraciones de eugenol en peces escalares (Pterophyllum scalare). Planos de recuperación: (a) Recuperación I, (b) Recuperación II, (c) Recuperación III, (d) Recuperación IV. Letras diferentes denotan diferencias estadísticas $(p<0.05)$ 


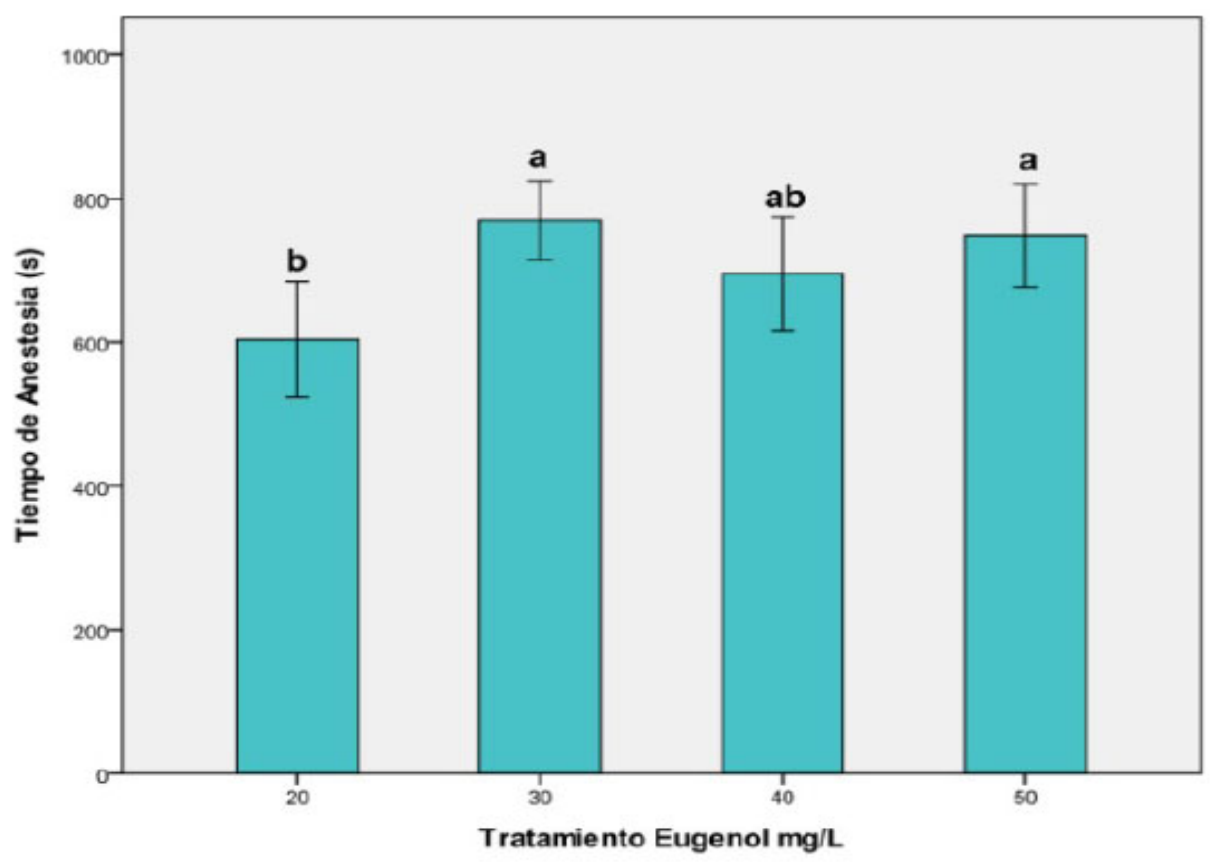

Figura 3. Tiempo total de anestesia (inducción y recuperación) a cuatro concentraciones de eugenol en peces escalares (Pterophyllum scalare). Se evidencian diferencias entre tratamientos $(p<0.05)$. La barra indica la desviación estándar.
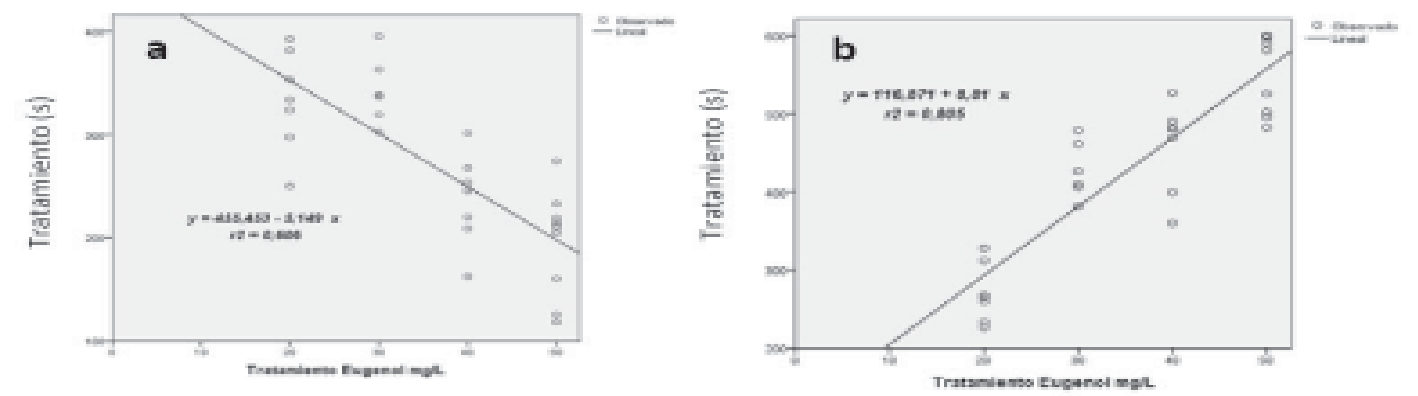

Figura 4. Tiempo total de inducción (a) y recuperación (b) a diferentes concentraciones de eugenol en peces escalares (Pterophyllum scalare). Regresión linear $(\mathrm{p}<0.05)$

No se observaron diferencias estadísticas entre tratamientos en el estadio de tiempo de recuperación; sin embargo, hubo diferencias estadísticas entre tratamientos $(\mathrm{p}<0.05)$ en los demás estadios, requiriendo menores tiempos de recuperación en las concentraciones más bajas (Fig. 2).
$\mathrm{Al}$ analizar el tiempo total de anestesia (inducción y recuperación; Fig. 3), la concentración de a $20 \mathrm{mg} / \mathrm{L}$ de eugenol registró el menor tiempo $(\mathrm{p}<0.05)$, aunque fue similar a la concentración de $40 \mathrm{mg} / \mathrm{L}$. Teniendo en cuenta los tiempos totales de inducción y recuperación para cada concentración, fue evi- 
dente una variación de los datos en forma lineal. Se presentó una disminución del tiempo de inducción $\left(\mathrm{R}^{2}=0.606, \mathrm{p}<0.05\right)$, así como un aumento en el tiempo de recuperación $\left(\mathrm{R}^{2}\right.$ $=0.805, \mathrm{p}<0.05)$ a medida que se aumentaba la concentración del anestésico (Fig. 4).

En los parámetros físico-químicos del agua no se evidenciaron diferencias ni variaciones significativas entre los grupos sometidos bajo las mismas condiciones experimentales por cada tratamiento. Tampoco se observó un efecto anestésico del alcohol diluyente.

\section{Discusión}

La ausencia de enfermedades y mortalidad en los individuos inducidos a anestesia con eugenol estuvo acorde con reportes previos (Guénette et al., 2007). Asimismo, la hiperactividad inicial durante la fase de inducción de la anestesia puede ser atribuida al propio eugenol ya que se ha descartado el efecto del alcohol diluyente (Mylonas et al., 2005).

El eugenol surge como una alternativa para la sedación y anestesia de peces, dado su reducido costo, escasa toxicidad y baja bioacumulación en los tejidos (Cooke et al., 2004; Kildea et al., 2004; Guénette et al., 2007); sin embargo, se dispone de reportes que señalan un bajo índice terapéutico (Iversen et al., 2003; Velísek et al., 2006) y resultados dependientes de la especie inducida a anestesia (Oliveira et al., 2008). Así, en salmónidos se ha encontrado que bajas concentraciones pueden ser tóxicas (Hussein et al., 2000), mientras que los carácidos presentan mayor tolerancia (Naranjo et al., 2010); asimismo, factores como temperatura, salinidad y conductividad pueden afectar el efecto del eugenol. Por otro lado, Hikasa et al. (1986) señalan que el periodo de recuperación es más corto a temperaturas altas, de allí la importancia de brindar atención especial a la temperatura del agua (Iversen $e t$ al., 2003).
Puede ocurrir mortalidad en peces con el empleo de eugenol si el tiempo de exposición o la concentración superan el margen de seguridad (Sladky et al., 2001); por ello, se debe tener en cuenta factores como la especie, la etapa del ciclo de vida en que se encuentra, la edad, tamaño y peso, contenido lipídico, condición corporal y estado de salud, pues estos factores afectan la tasa metabólica; además de cambios provocados en la captación de oxígeno a través de las branquias y el incremento o disminución de la eficiencia del agente anestésico (Burka et al., 1997).

Velísek et al. (2007) definieron tres criterios que debe cumplir un anestésico aplicado en acuicultura: debe ser efectivo, seguro y económico. La eficacia se consigue cuando la sedación se debe lograr en un periodo menor o igual a tres minutos y la recuperación del nado normal en un periodo no mayor a 10 minutos (Oliveira et al., 2007, 2008). Bajo estos parámetros, la concentración anestésica más efectiva fue de $40 \mathrm{mg} / \mathrm{L}$, acorde con lo sugerido por Griffits (2000) y cercano a las recomendaciones de $30 \mathrm{mg} / \mathrm{L}$ de Prince y Powell (2000) en truchas arcoiris y de Svoboda y Koláøva (1999). Concentraciones más elevadas han sido reportadas en percas plateadas (Bidyanus bidyanus) donde con $50 \mathrm{mg} / \mathrm{L}$ perdían el eje de nado y el equilibrio, entrando en una plano medio de anestesia (Kildea et al., 2004), así como de $100 \mathrm{mg} / \mathrm{L}$ en Siganus lineatus (Soto y Burhanuddin, 1995) y 100 y $120 \mathrm{mg} / \mathrm{L}$ para trucha arco iris (Anderson et al., 1997). De la misma manera, Akbulut et al. (2011) demostraron en el esturión ruso (Acipenser gueldenstaedtii), que concentraciones mayores de $200 \mathrm{mg} / \mathrm{L}$ y hasta $750 \mathrm{mg} / \mathrm{L}$ poseen un efecto anestésico acorde con los parámetros de anestesia óptimos para peces. Por otro lado, Briozzo et al. (1989) reportan bajas concentraciones anestésicas (5 a 8.5 $\mathrm{mg} / \mathrm{L}$ ) para el manejo y transporte de Micropterus salmoides.

La concentración de $20 \mathrm{mg} / \mathrm{L}$ induce una ligera anestesia que permitiría realizar 
procedimientos tales como morfometría, pesaje, marcación, transporte, clasificación, etc. De la misma manera, la concentración de $50 \mathrm{mg} / \mathrm{L}$ permitiría procedimientos de mayor complejidad, incluyendo cirugías o procedimientos invasivos (como biopsias).

El menor tiempo de recuperación se encontró con la concentración de $20 \mathrm{mg} / \mathrm{L}$, resultado que concuerda con los hallazgos de Cooke et al. (2004), quienes describieron que el mayor plano de profundidad de anestesia incrementaba significativamente al aumentar la concentración de eugenol $\left(\mathrm{R}^{2}=0.85\right.$, $\mathrm{p}<0.001$ ), así como el registro de mayores tiempos en la recuperación cuando los animales alcanzan tales estados profundos de anestesia. Asimismo, peces en estado 3 de anestesia requirieron entre 10 a $30 \mathrm{~min}$ de recuperación (Velísek et al., 2006). Estos resultados pueden entenderse a partir de los mecanismos de la farmacocinética del eugenol, el cual a una concentración de 75 $\mathrm{mg} / \mathrm{L}$ presenta una vida media de 12.1 horas en la sangre, lo que sugiere una acumulación en el organismo dado el caso que se requieran repetidas administraciones. La curva bifásica sugiere una circulación entero-hepática, característica de los componentes fenoles sometidos a la conjugación, contribuyendo al mayor tiempo de eliminación (Guénnete et al., 2007).

El efecto anestésico y analgésico del eugenol ha sido atribuido a su acción inhibitoria sobre los canales de sodio (Park et al., 2006) y calcio, especialmente los canales tipo N-calcio o High voltage activated calcium channel (HVACC), dado que bloquea la generación del potencial de acción y produce una disminución en la liberación de neurotransmisores dependientes de calcio en las terminaciones presinápticas neuronales (Lee et al., 2005). Estudios in vitro han demostrado el efecto bloqueante del eugenol sobre los receptores vaniloides (TRPV1Transient receptor potential vanilloid 1), inhibiendo así la transmisión del dolor (Yang et al., 2003); sin embargo, Lee et al. (2005) indican que la acción del eugenol no es de- pendiente de TRPV1. Ohkubo y Kitamura (1997) reportan que el eugenol puede activar los receptores de capsaicina, y sugieren, además, que el eugenol posee una acción de tipo específica, mediante la inducción de corriente en la membrana de las neuronas sensoriales y también puede activar de forma reversible canales permeables al $\mathrm{Ca}^{2+}$ y canales $\mathrm{de}^{-}$dependientes de calcio. Adicionalmente, a este anestésico se le ha atribuido el incremento en la liberación de sustancia P de las neuronas nociceptivas polimodales (Bevan y Szolcsányi, 1990). Otros autores sustentan que el efecto analgésico del eugenol está directamente mediado por la inhibición de la síntesis de prostaglandinas y leucotrienos (Dohi et al., 1991).

De igual manera, se ha descrito que el isoeugenol, componente del eugenol, bloquea la transmisión de la información sensorial al hipotálamo a partir de la interacción con neurotransmisores relacionados con la sensibilidad al dolor, el efecto agonista en GABA (ácido gamma-amino butírico) y antagonista de glutamato (NMDA) (Aoshima y Hamamoto, 1999; Yang et al., 2003).

\section{Conclusiones}

- El eugenol (aceite de clavo) resulta ser una herramienta anestésica importante en peces escalares (Pterophyllum scalare), ya que los animales alcanzan adecuada inducción y recuperación, garantizando así la inocuidad del anestésico.

- La concentración anestésica más efectiva fue de $40 \mathrm{mg} / \mathrm{L}$ de eugenol, debido a que se alcanza en menor tiempo (4 minutos) estadios de anestesia 3 con periodos de recuperación total equivalentes a 7 minutos.

\section{LiTERATURA Citada}

1. Ackerman J, Bellwood D. 2002. Comparative efficiency of clove oil and 
rotenone for sampling tropical reef fish assemblages. J Fish Biol 60: 893-901.

2. Akbulut B, Çavdar Y, Çakmak E, Aksungur N. 2011. Use of clove oil to anaesthetize larvae of Russian sturgeon (Acipenser gueldenstaedtii). J Appl Ichthyol 27: 618-621.

3. Anderson WG, McKinley RS, Colavecchia M. 1997. The use of clove oil as an anaesthetic for rainbow trout and its effects on swimming performance. N Am J Fish Manage 17: 301-307.

4. Aoshima H, Hamamoto K. 1999. Potentiation of GABA A receptors expressed in Xenopus oocytes by perfumes and phytoncid. Biosci Biotechnol Biochem 63: 743-748.

5. Bandell M, Story G, Hwang W, Viswanath V, Eid S, Petrus M, et al. 2004. Noxious cold ion channel TRPA1 is activated by pungent compounds and bradykinin. Neuron 41: 849-857.

6. Bevan S, Szolcsányi J. 1990. Sensory neuron-specific actions of capsaicin: mechanisms and applications. Trends Pharmacol Sci 11: 330-333.

7. Briozzo J, Nunez L, Chirife $J$, Herszage L, D’Aquino M. 1989. Antimicrobial activity of clove oil dispersed in a concentrated sugar solution. J Appl Bacteriol 66: 69-75.

8. Burka JF, Hammel KL, Horsberg TE, Johnson GR, Rainnie DJ, Spears DJ. 1997. Drugs in salmonid aquaculture - a review. J Vet Pharmacol Ther 20: 333-349.

9. Cooke SJ, Suski CD, Ostrand KG, Tufts BL, Wahl DH. 2004. Behavioral and physiological assessment of low concentrations of clove oil anaesthetic for handling and transporting largemouth bass (Micropterus salmoides). Aquaculture 239: 509-529.

10. Coyle S, Durborow R, Tidwell J. 2004. Anesthetics in aquaculture. Southern Regional Aquaculture Center Publication N. ${ }^{\circ}$ 3900. Texas, USA. 6 p.

11. Cunha F, Rosa I. 2006. Anaesthetic effects of clove oil on seven species of tropical reef teleosts. J Fish Biol 69: 15041512.
12. Dohi T, Anamura S, Shirakawa M, Okamoto H, Tsujimoto A. 1991. Inhibition of lipoxygenase by phenolic compounds. Jpn J Pharmacol 121: 146147.

13. Façanha M, Gomes L. 2005. A eficácia do mentol como anestésico para tambaqui (Colossoma macropomum, Characiformes: Characidae). Acta Amazonica 35: 71-75.

14. Griffiths SP. 2000. The use of clove oil as an anaesthetic and method for sampling intertidal rockpool fishes. J Fish Biol 57: 1453-1464.

15. Guénette S, Uhland F, Hélie P, Beaudry F, Vachon P. 2007. Pharmacokinetics of eugenol in rainbow trout (Onchorhynchus mykiss). Aquaculture 266: 262-265.

16. Harris B. 2006. Menthol: A review of its thermoreceptor interactions and their therapeutic applications. Internat $\mathrm{J}$ Aromatherapy 16: 117-131.

17. Hikasa Y, Takase K, Ogasawara T, Ogasawara S. 1986. Anesthesia and recovery with tricaine methanesulfonate, eugenol and thiopental sodium in the carp, Cyprinus carpio. Jpn J Vet Sci 48: 341-351.

18. Hill J, Forster M. 2004. Cardiovascular responses of Chinook salmon (Oncorhynchus tshawytscha) during rapid anaesthetic induction and recovery. Comp Biochem Physiol 137: 167-177.

19. Hussein MM, Wada S, Hatai K, Yamamoto A. 2000. Actimycotic activity of eugenol against selected water molds. J Aqua Anim Hlth 12: 224-229.

20. Iversen M, Finstand B, Mackinley $R$, Eliassen R. 2003. The efficacy of metomidate, clove oil, Aqui-S and Benzoak $^{\circledR}$ as anaesthetics in Atlantic salmon (Salmo salar L.) smolts, and their potential stress-reducing capacity. Aquaculture 221: 549-566.

21. Keene J, Noakes D, Moccia R, Soto C. 1998. The efficacy of clove oil as an anaesthetic for rainbow trout, Oncorhynchus mykiss (Walbaum). Aquaculture Res 29: 89-101. 
22. Kildea MA, Allan GL, Kearney RE. 2004. Accumulation and clearance of the anaesthetics clove oil and AQUI-STM from the edible tissue of silver perch (Bidyanus bidyanus). Aquaculture 232: 265-277

23. Lee M, Yeon K, Park C, Li H, Fang Z, Kim MS, Choi SY, et al. 2005. Eugenol inhibits calcium currents in dental afferent neurons. J Dent Res 84: 848-851.

24. Molinero A, Gonzalez J. 1995. Comparative effects of MS 222 and 2phenoxyethanol on gilthead sea bream (Sparus aurata L.) during confinement. Comp Biochem Physiol 111: 405-414.

25. Munday P, Wilson S. 1997. Comparative efficacy of clove oil and other chemicals in anaesthetization of Pomacentrus amboinensis, a coral reef fish. J Fish Biol 51: 931-938.

26. Mylonas C, Cardinaletti T, Sigelaki I, Polzonetti-Magni A. 2005. Comparative efficacy of clove oil and 2phenoxyethanol as anesthetics in the aquaculture of European sea bass (Dicentrarchus labrax) and gilthead sea bream (Sparus aurata) at different temperatures. Aquaculture 246: 467-481.

27. Naranjo L, Marín G, Chacón R, Hernández T, Torres A, Rondón I. 2010. Ensayos preliminares de la concentración letal 50 y dosis efectiva anestésica del eugenol en cachama blanca (Piaractus brachypomus). En: Memorias XVI Jornada de Acuicultura IALL. Villavicencio, Meta: Universidad de Los Llanos. p 134-137.

28. Ohkubo T, Kitamura K. 1997. Eugenol activates $\mathrm{Ca}^{+2}$ permeable currents in rat dorsal root ganglion cells. J Dent Res 76: 1737-1744.

29. Oliveira L, Branco R, Luscher A, Lira A, Rocha T, Santos G. 2008. Eugenol como anestésico para a tilápia-do-nilo. Pesq Agropec Bras 43: 1069-1074.

30. Oliveira LV, Massamitu W, Sakaguti T, Rodrigues C, Dena dos Santos L, Martins C. 2007. Concentrações de Eugenol para anestesia profunda e toxidade aguda em juvenis de piavuçu (Leporinus macrocephalus). Acta Sci 29: 357-362.

31. Park C, Ki H, Yeon K, Jung S, Choi S, Lee SJ, Lee S, et al. 2006. Eugenol inhibits sodium currents in dental afferent neurons. J Dent Res 85: 900-904.

32. Prince A, Powell C. 2000. Clove oil as an anaesthetic for invasine field procedures on adult rainbow trout. N Am Fish Manage 20: 1029-1032.

33. Seol D, Lee J, Im S, Park I. 2007. Clove oil as an anaesthetic for common octopus (Octopus minor, Sasaki). Aquaculture Res 38: 45-49.

34. Sladky KK, Swanson CR, Stoskopf MK, Loomis MR, Lewbart GA. 2001. Comparative efficacy of tricaine methasulfonate and clove oil for use as anesthetics in red pacu (Piaractus brachypomus). Am J Vet Res 62: 337342.

35. Soltani M, Marmari G, Mehrabi M. 2004. Acute toxicity and anesthetic effects of clove oil in Penaeus semisulcatus under various water quality Conditions. Aquacul Int 12: 457-466.

36. Soto C, Burhanuddin. 1995. Clove oil as a fish anaesthetic for measuring length and weight of rabbitfish (Siganus lineatus). Aquaculture 136: 149-152.

37. Svoboda M, Koláøova J. 1999. A survey of anaesthetics used in the fish farming [in Czech]. Proc of Health Protection of Fish. Research Institute of Fish Culture and Hydrobiology, Vodòany, Czech Republic.

38. Tort L, Puigcerver $M$, Crespo S, Padrós F. 2002. Cortisol and haematological response in sea bream and trout subject to the anaesthetic clove oil and 2-phenoxyethanol. Aquac Res 33: 907-910.

39. Velísek J, Wlasow T, Gomulka P, Svobodová Z, Novotn L, Ziomek E. 2006. Effects of clove oil anaesthesia on European Catfish (Silurus glanis L.). Acta Vet Brno 75: 99-106. 
40. Velisek J, Wlasow T, Gomulka P, Svobodova Z, Novotny L. 2007. Effects of 2-phenoxyethanol anaesthesia on sheatfish (Silurus glanis L.). Vet MedCzech 52: 103-110.

41. Walsh CT, Pease BC. 2002. The use of clove oil as an anaesthetic for the longfinned eel, Anguilla reinhardtii (Steindachner). Aquac Res 33: 627-635.
42. Woody C, Nelson J, Ramstad K. 2002. Clove oil as an anaesthetic for adult sockeye salmon: field trials. J Fish Biol 60: 340-347.

43. Yang BH, Piao ZG, Kim YB, Lee CH, Park K, Kim JS, Oh SB. 2003. Activation of vanilloid receptor 1 (VR1) by eugenol. J Dent Res 82: 781-785. 